\title{
Patient satisfaction: an indicator of quality in disablement services centres
}

\author{
C Smith, M McCreadie, J Unsworth, H I Wickings, A Harrison
}

\begin{abstract}
Objectives-To develop a patient satisfaction system for disablement services centres and to report on how the initial findings have been used in audit to improve their quality of care and services.

Design-Interview survey of randomly selected users attending in three centres: Birmingham (centre $\mathbf{X}$ ), Oxford (centre $Y$ ), and Cambridge (centre $Z$ ) to establish core topics for developing a patient satisfaction questionnaire with incorporation into a computer patient satisfaction system (PATSAT) to enable collation of responses to the questionnaire. A pilot of the questionnaire was undertaken in the centres to assess the sensitivity of the questionnaire, which was subsequently used as part of clinical audit process during June 1991 and April 1992 in centre $X$ and the patient satisfaction system used to monitor changes in routine practice.

Patients-123 amputees in the development phase, selected by cluster sampling, and 1103 amputees in the pilot study.

Main measures-Satisfaction scores for components of the service.

Results-The questionnaire included 16 core topics contributing to quality of care and services, including comfort of limbs, appointments, interpersonal aspects of care, a system of support and counselling, and organisation. The pilot survey demonstrated high satisfaction scores for aspects of interpersonal care, organisation, and physical surroundings of the centres and lower satisfaction for counselling services, comfort of the limb and the number of alterations made before the limb was considered acceptable. During the audit in centre $X$ these results prompted changes to care and services which produced significant improvements in satisfaction. Conclusions-The early results suggest that the questionnaire, coupled with PATSAT software system, enable users' views to be expressed, collated, and fed back to staff; the information provided has already prompted change, and the system is sufficiently sensitive to measure changes in satisfaction with the service. (Quality in Health Care 1995;4:31-36)
\end{abstract}

Keywords: patient satisfaction, quality indicator, disablement services, audit, amputees.

\section{Introduction}

Measures of patient satisfaction are being used increasingly as part of the assessment of the quality of care and they are being incorporated into the process of clinical audit. Satisfaction is an important component of the total assessment of the value of treatment and care. Satisfaction with care is an important influence determining whether a person seeks medical advice, complies with treatment, and maintains a relationship with the practitioner. ${ }^{1}$ For users of artificial limbs the difficulties that result from dissatisfaction with services may be profound, and any alternative accessible help is unlikely.

Several studies have examined patient satisfaction within the artificial limb service. None has attempted to develop a system to assess satisfaction routinely or to integrate information about satisfaction into the process of clinical audit. This paper describes the development of a patient satisfaction system developed for disablement services centres, reports how the initial findings were used in an audit to improve the quality of care and services provided to patients, and shows the effect of incorporating users' views into the clinical audit of prosthetic services.

\section{Methods}

The study was done at three disablement services centres: Birmingham (centre $\mathrm{X}$ ), one of the largest centres in the country, with about 5000 current prosthetic patients; Oxford (centre Y), with about 2500 current attenders; and Cambridge (centre $\mathrm{Z}$ ), one of the smaller centres, with about 1700 patients. The services provided by the three centres vary, as do the resources available at each.

DEVELOPMENT OF QUESTIONNAIRE

We developed a patient satisfaction questionnaire based on issues relevant to users of the service. Users of artificial limbs and new amputees attending clinics in the three centres were selected randomly by cluster sampling, and the selected 123 patients were interviewed at the centre after their clinic appointment. The purpose of the study was explained by a nurse at the centre, who confirmed users' willingness to take part. Semistructured interviews were carried out by interviewers from CASPE Research, and the interviewees were asked to identify those components of care and services which influenced their satisfaction.

In addition, the views of managers, doctors, the prosthetist, and other clinicians were obtained to identify the issues the staff considered important for inclusion in the questionnaire. These interviewees were 
selected by purposive sampling, and interviews were carried out using a different semistructured interview schedule.

Data from the interviews were ranked to identify the topics that interviewees identified as being important to their satisfaction and these were ranked in order of frequency. All data were entered on to a microcomputer, using general purpose database software. Thus core topics for the questionnaire were identified and questions were designed to explore each topic in the questionnaire. In addition to the core topics, each centre was able to select up to eight topics which reflected local staff concerns. The questions were designed to be short and unambiguous.

Once the questionnaire had been constructed convenience sampling was carried out at the three centres, and a further 50 service users were selected and interviewed to confirm the content validity of the questions, by examining their interpretation of each question. For questions scoring less than $85 \%$ agreement on content, the question was reworded and retested. Where there were problems in interpreting questions a detailed content analysis was carried out, by examining the content of words or descriptions within a predefined coded category.

Each question was posed on a 4 point scale: very satisfied, satisfied, somewhat dissatisfied, and very dissatisfied, and scores were allocated across a Likert scale ( 1 very dissatisfied, 4 very satisfied). Satisfaction indices were then calculated for each topic and each respondent and the total score divided by the maximum possible score. The range of scores across the index of satisfaction was $0 \cdot 25$ (very dissatisfied) to 1.0 (very satisfied). In this way indices of satisfaction can be produced for any topic. The appropriateness of this scale has been assessed previously. ${ }^{23}$ Additional research has shown such indices are sufficiently sensitive to detect changes in satisfaction at local levels, such as in wards or departments. ${ }^{4-6}$ Space on

\section{Core topics identified from pilot}

1 Surroundings in the centre

2. Facilities while waiting

3. Friendliness and politeness of the doctors

4. Friendliness and politeness of the prosthetist (fitter)

5. Waiting time in the prosthetist's (fitting) room

6. Kindness and help given to you by staff

7. Staff's willingness to talk and listen to you

8. Availability of information about your limb, fitting, aftercare

9. Availability of counselling and support services

10. Skill of the prosthetist (fitter) with fitting your limb

11. Comfort of your limb

12. Usefulness of your limb for daily activities

13. Number of alterations to your limb before it was acceptable

14. Arrangements for booking your appointments

15. Layout of the centre

16. Overall way the centre is organised the questionnaire was also available for additional comments, which were analysed by a text and retrieval package which reports on topics relating to, for example, limb comfort.

\section{COMPUTER PROGRAMMING}

The second phase of development entailed programming the computers. The patient satisfaction system being developed here (PATSAT) and the disablement services centre database Limbsys (the management information system) were made compatible, enabling users' satisfaction scores to be linked to information on aspects of service delivery such as the doctor, prosthetist, or site of amputation. This facility allows assessment of satisfaction for each core topic according to type and level of amputation, age and sex of respondent, and the prosthetic contractor. This level of analysis is not reported in this paper, although it is routinely produced for users of the PATSAT system, allowing comparisons to be made. In addition, the respondents' verbatim comments can be linked to satisfaction scores through search and retrieval text software.

PILOT OF PATIENT SATISFACTION SYSTEM FOR USERS OF DISABLEMENT SERVICES CENTRES Once the questionnaire and software had been developed, the system for monitoring patient satisfaction was piloted in three centres for six months from March 1991. Every patient visiting the centre received a personalised letter and questionnaire from reception staff. Patients were identified from the appointment system, which interacted with the PATSAT software and identified their eligibility to take part in the study. Those who had received a questionnaire in the previous six months were excluded from the study. Patients were encouraged to complete their questionnaire on site, and at the end of their appointment. For the pilot study the responders completed questionnaires at various stages in the process of obtaining a limb. Non-responders were not followed up.

FEEDBACK OF RESULTS AND MONITORING CHANGES IN ROUTINE PRACTICE

Data were processed routinely by CASPE Research and reports were generated monthly and disseminated to clinical and managerial staff in the centres.

After introduction of the patient satisfaction system we examined whether the system could trigger improvements in the quality of care. Accordingly, in centre X (Birmingham) satisfaction scores were analysed in June 1991 and were compared nine months later, in April 1992. In June 1991, 160 amputees completed the questionnaire and in April 1992, 114 did so. Once again users could re-enter the study only every six months, therefore some of the respondents had an opportunity to comment twice.

STATISTICAL ANALYSIS

Between the two periods the percentage of satisfied respondents was compared. Owing 
Table 1 Response rate by sex and age group for three disablement services centres. Figures are perecentages (numbers)

\begin{tabular}{|c|c|c|c|c|c|c|c|c|c|}
\hline & \multicolumn{3}{|c|}{$\begin{array}{c}\text { Centre } X \\
(n=1155)\end{array}$} & \multicolumn{3}{|c|}{$\begin{array}{l}\text { Centre } Y \\
(n=566)\end{array}$} & \multicolumn{3}{|c|}{$\begin{array}{c}\text { Centre } Z \\
(n=222)\end{array}$} \\
\hline & Responders & Non-responders & Total ${ }^{\star}$ & Responders & Non-responders & Total ${ }^{\star}$ & Responders & Non-responders & Total ${ }^{\star}$ \\
\hline \multicolumn{10}{|l|}{ Sex } \\
\hline Male & $62 \cdot 6(508)$ & $37 \cdot 4(303)$ & 811 & $48 \cdot 2(196)$ & $51 \cdot 8(211)$ & 407 & $51.9(84)$ & $48 \cdot 1(78)$ & 162 \\
\hline Female & $63 \cdot 1(217)$ & $36.9(127)$ & 344 & $43 \cdot 4(89)$ & $56 \cdot 6(90)$ & 159 & $50 \cdot 9(29)$ & $49 \cdot 1(28)$ & 57 \\
\hline \multicolumn{10}{|l|}{ Age (years): } \\
\hline $10-19$ & $72 \cdot 4(21)$ & $27.6(8)$ & 29 & $\star \star$ & $\star \star$ & 15 & $\star \star$ & $\star \star$ & 3 \\
\hline $20-29$ & $57 \cdot 9(44)$ & $42 \cdot 1(32)$ & 76 & $42 \cdot 6(20)$ & $57 \cdot 4(27)$ & 47 & $41 \cdot 7(10)$ & $58 \cdot 3(14)$ & 24 \\
\hline $30-39$ & $62 \cdot 9(66)$ & $37 \cdot 1(39)$ & 105 & $51 \cdot 9(28)$ & $48 \cdot 1(26)$ & 54 & $\star \star$ & $\star \star$ & 10 \\
\hline $40-49$ & $55 \cdot 2(69)$ & $44 \cdot 8(56)$ & 125 & $53 \cdot 1(34)$ & $46.9(30)$ & 64 & $35 \cdot 3(12)$ & $64 \cdot 7(22)$ & 34 \\
\hline $50-59$ & $64 \cdot 6(102)$ & $35 \cdot 4(56)$ & 158 & $52 \cdot 4(44)$ & $47 \cdot 6(40)$ & 84 & $61 \cdot 5(16)$ & $38 \cdot 5(10)$ & 26 \\
\hline $60-69$ & $64 \cdot 4(163)$ & $35.6(90)$ & 253 & $52 \cdot 0(52)$ & $48 \cdot 0(48)$ & 100 & $64 \cdot 6(31)$ & $35 \cdot 4(17)$ & 48 \\
\hline $70-79$ & $66 \cdot 7(174)$ & $33 \cdot 3(87)$ & 261 & $48 \cdot 0(61)$ & $52 \cdot 0(66)$ & 127 & $61 \cdot 1(33)$ & $38.9(21)$ & 54 \\
\hline $80-89$ & $63 \cdot 2(72)$ & $36 \cdot 8(42)$ & 114 & $29 \cdot 6(21)$ & $70 \cdot 4(50)$ & 71 & $40.0(8)$ & $60 \cdot 0(12)$ & 20 \\
\hline
\end{tabular}

${ }^{\star}$ Missing data in each case.

$\star \star D$ ata for $<20$ cases.

Centre $X\left(X^{2}=7 \cdot 16, d f=7 ; p>0.05\right)$

Centre $Y\left(X^{2}=11.59, \mathrm{df}=7 ; \mathrm{p}>0.05\right)$
Centre $Z\left(\chi^{2}=15.67, \mathrm{df}=5 ; 0.01<\mathrm{p}<0.05\right)$.

to the limited ability of the satisfaction scale to discriminate between categories with small numbers, the scale was reduced to a 2 point scale measuring, respectively, the percentages of responders who were satisfied and of those who were dissatisfied. ${ }^{2}$ A test between sample proportions was used to examine differences between the proportions of users satisfied between June 1991 and April 1992.

In all three centres the characteristics of responders and non-responders were examined. A test between sample proportions was used to examine differences in responses between men and women and a $\chi^{2}$ test to examine differences in age between responders and non-responders. Non-parametric tests were used to determine significance for data with non-normal distributions.

\section{Results}

DEVELOPMENT OF QUESTIONNAIRE

Over 40 topics were described as important to satisfaction by limb amputees during the development phase of the questionnaire, which included: comfort in wearing the limb, appointments, interpersonal aspects of care, a system of support and counselling, and aspects of organisation and efficiency of running of the centres. The rank order of topics (box) determined the content of the questionnaire, the 16 topics forming the core questions. No appreciable difference in ranking was evident between the three centres. Content validity of the questionnaire was found to be high: one question, concerning the perceptions of organisation of the centre, scored less than

Table 2 Response rate by level of amputation for two centres. Figures are percentages (numbers)

\begin{tabular}{|c|c|c|c|c|c|c|}
\hline \multirow[b]{2}{*}{ Site of amputation } & \multicolumn{3}{|c|}{ Centre $X$} & \multicolumn{3}{|c|}{ Centre $Y$} \\
\hline & Responders & Non-responders & Total & Responders & Non-responders & Total \\
\hline Lower limb below knee & $65 \cdot 2(375)$ & $34 \cdot 8(200)$ & 575 & $48 \cdot 5(159)$ & $51 \cdot 5(169)$ & 328 \\
\hline Lower limb above knee & $67 \cdot 1(192)$ & $32 \cdot 9(94)$ & 286 & $44 \cdot 0(55)$ & $56 \cdot 0(70)$ & 125 \\
\hline Upper limb below elbow & $42 \cdot 9(21)$ & $57 \cdot 1(28)$ & 49 & $\star \star$ & $\star \star$ & 9 \\
\hline Upper limb above elbow & $42 \cdot 9(12)$ & $57 \cdot 1(16)$ & 28 & $\star \star$ & $\star \star$ & 13 \\
\hline Congenital amputation & $40 \cdot 0(12)$ & $60 \cdot 0(18)$ & 30 & $\star \star$ & $\star \star$ & 8 \\
\hline Multiple amputation & $59 \cdot 0(46)$ & $41 \cdot 0(32)$ & 78 & $74 \cdot 3(29)$ & $25 \cdot 6(10)$ & 39 \\
\hline
\end{tabular}

$\star \star$ Data for $<20$ cases.

Site $X: \chi^{2}=20 \cdot 15, \mathrm{df}=5 ; \mathrm{p}<0 \cdot 05$.
$85 \%$ agreement and was reworded to "the overall way the centre is organised."

PILOT OF PATIENT SATISFACTION SYSTEM

In the six month pilot study a total of 1943 amputees were sent questionnaires and asked to take part; 1103 responded, giving a response rate of $56 \cdot 8 \%$. For each centre the number of responders was $62 \cdot 8 \%(725 / 1155$, centre $\mathrm{X})$ $46.8 \%(265 / 566$, centre $Y)$, and $50.9 \%$ (113/222, centre Z).

Tables 1 and 2 show characteristics of responders and non-responders. No difference in the response rate between men and women was found. The age of responders and nonresponders was similar in two centres ( $\mathrm{X}$ and Y). A $\chi^{2}$ test of the data within each centre showed a significant difference in age between responders and non-responders only in centre $\mathrm{Z}$, in which non-responders tended to be younger than the responders.

Over $70 \%(1380 / 1943)$ of users of the disablement services were male (table 1), and use of the services increased above the 50-59 age group, with the greatest proportional use among the 70-79 age group. Data on site of amputation could be extracted only for centres $\mathrm{X}$ and $\mathrm{Y}$ (table 2). Amputations of the lower limb were the most common amputation in centres $\mathrm{X}$ and $\mathrm{Y}$, and within this category there was a greater proportion of below knee amputations ( $66 \cdot 7 \%$ and $72 \cdot 4 \%$ respectively). In centre $\mathrm{X}$ a significant difference in response rate was found between upper and lower limb amputees. Too few data were available for centre $\mathrm{Y}$ to enable analysis.

\section{SATISFACTION INDICES}

Figure 1 shows the satisfaction indices for all topics for the three centres. A score of 1.0 represents a response of "very satisfied" and 0.25 "very dissatisfied." For all three centres user satisfaction was high $(0.80-0.90)$ or higher for surroundings, layout, booking of appointments, waiting facilities, and aspects of interpersonal care such as staff help and the attitudes of doctors and prosthetists, and limb fitting. Four main topics scored lower: waiting time in the fitting room, availability of 


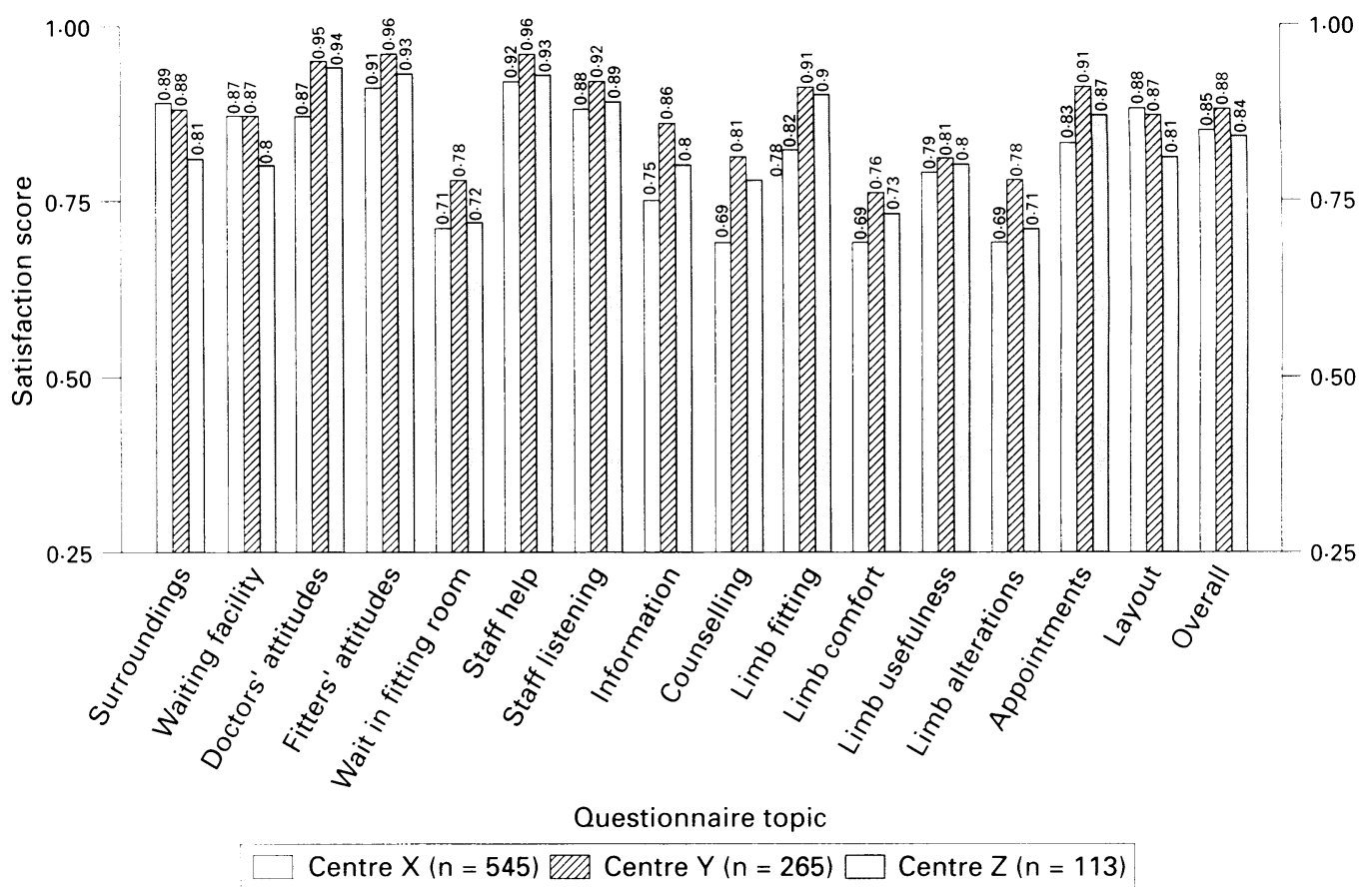

Fig 1 Patients' satisfaction with main aspects of care in disability services centres, March and August 1991

\section{Changes made by Birmingham centre} to improve service

- Information folder on disability

- Publicity on the information department

- Nursing auxiliaries reassigned and given new responsibilities

- Fitting room made available for couples

- Introduction of an outdoor test track

- Funding to employ a social counsellor

counselling services, comfort of the limb, and the number of alterations before the limb was considered to be acceptable.

AUDIT AT BIRMINGHAM DISABLEMENT SERVICES CENTRE

Each centre had the opportunity to assess and use information from the patients' views of its care and services. The results from the Birmingham centre were chosen to illustrate how the results from the routine use of the questionnaire linked to PATSAT software were used in practice. Satisfaction scores for services provided by the Birmingham centre (X in fig 1) indicated satisfaction with aspects of service delivery and many of the interpersonal aspects of care. Lower levels of satisfaction compared with the two other centres were visually apparent (fig 1 ) for waiting time in the fitting room, information, counselling, fitting

Table 3 Comparison of users' satisfaction with selected aspects of services at Birmingham after changes in practice

\begin{tabular}{llcr}
\hline \multirow{2}{*}{ Topic } & \multicolumn{3}{c}{$\%$ No of patients satisfied } \\
\cline { 2 - 4 } & \multicolumn{1}{c}{ fune 1991} & \multicolumn{1}{c}{ April 1992} & Significance $^{\star}$ \\
\hline Information & $70(106 / 152)$ & $87(90 / 104)$ & $\mathrm{p}<0 \cdot 01$ \\
Counselling & $67(85 / 127)$ & $81(84 / 104)$ & $\mathrm{p}<0 \cdot 01$ \\
Fitting of limb & $85(126 / 148)$ & $91(95 / 104)$ & $0 \cdot 01<\mathrm{p}<0 \cdot 05$ \\
Comfort of limb & $57(85 / 149)$ & $74(81 / 110)$ & $\mathrm{p}<0 \cdot 01$ \\
Usefulness of limb & $80(116 / 146)$ & $84(92 / 109)$ & $\mathrm{p}>0 \cdot 05$ \\
Alterations to limb & $67(95 / 141)$ & $80(82 / 102)$ & $0 \cdot 01<\mathrm{p}<0 \cdot 05$ \\
Booking appointments & $84(127 / 152)$ & $92(100 / 109)$ & $\mathrm{p}<0 \cdot 01$ \\
\hline
\end{tabular}

*Test between sample proportions. of the limb, comfort of the limb, and the number of alterations before the limb was acceptable.

These results were reviewed in the Birmingham centre throughout the audit process during 1991, and in response several changes were introduced to improve the quality of care and services (box). Most artificial limbs are prescribed in an environment which does not offer the range of different surfaces on which the limb might be used. An outdoor track where users can test out their artificial limbs in more realistic conditions, such as on grass and slopes has been introduced.

Through the clinical audit process the comfort and usefulness of limbs and the number of alterations to limbs were highlighted as topics of major concern to users. As a result at the initial appointment the amputee, prosthetist, and healthcare professional now agree on a realistic outcome as a result of providing a prosthesis. The amputee's progress is monitored against this goal throughout the period of aftercare. In addition, the basis of contracts with the manufacturers of prostheses has been changed to reflect the need to achieve improved quality of care and user satisfaction through fewer visits to the centre and a more comfortable limb fitting. This will continue to be monitored and discussed every month with the contractors, according to feedback received on user satisfaction.

MONITORING CHANGES IN PRACTICE

To examine whether the changes introduced in the Birmingham centre led to improved satisfaction and quality of care, satisfaction recorded in June 1991 was compared with that in April 1992 for seven selected topics. The response rate in June 1991 was $64 \%$ and in April 1992, 53\%. Table 3 shows that for each selected core topic, except for usefulness of the 


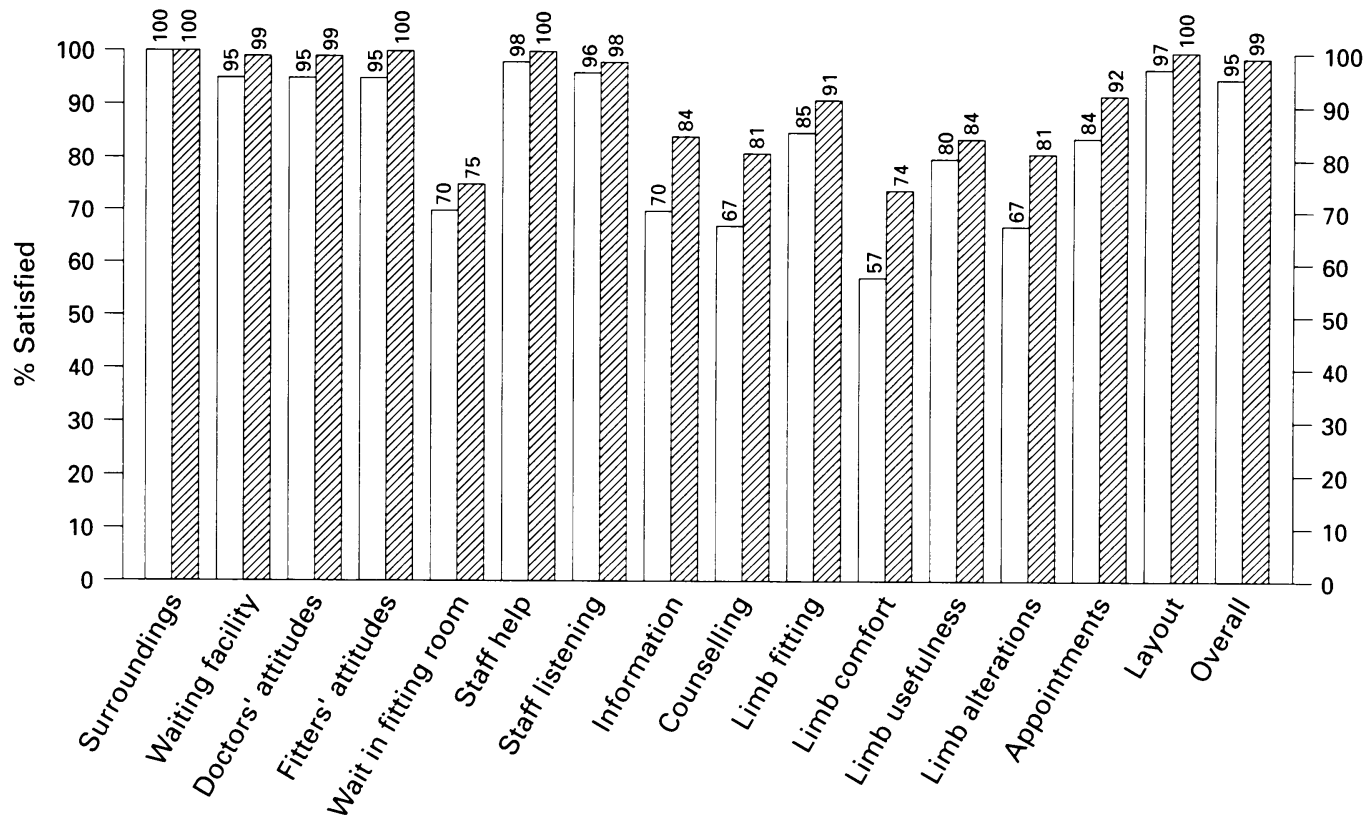

Questionnaire topic

June $1991(n=160)$ UIA April $1992(n=114)$

Fig 2 Patients' satisfaction with main aspects of care in Birmingham (centre X) in fune 1991 and April 1992

limb, the percentage of satisfied respondents in April 1992 had increased significantly and the improvements were evident across the entire satisfaction scale (fig 2)

\section{Discussion}

Early results from the use of this questionnaire of users' satisfaction with the service provided by three disablement services centres show that this tool is successful in enabling users to express their opinion of the service standards they receive. The system was sufficiently sensitive to measure variations in satisfaction with the service, it provided insights into areas of satisfaction and dissatisfaction with the provision of artificial limbs, and it highlighted some important differences between the centres. Feeding back this information to the centres has resulted in changes in service delivery whose effect can be measured by changes in satisfaction indices.

The questionnaire was developed by ranking topics raised by responders during the initial interviews. The actual rankings and satisfaction scores for each individual topic were different, and this highlights a conceptual difference between the two issues. To improve on future questionnaire design, incorporating a weighting factor, such as the degree of importance, during the initial rankings might help to identify the relative importance of the core questions. This would facilitate a subsequent review of satisfaction levels in audit and planning of proposed action.

The response rates were low in the pilot study, for reasons that are not clear. Only one set of questionnaires was sent out, and nonresponders were not followed up, owing to a lack of resources. Among the respondents upper limb amputees were underrepresented, which is likely to be related to their physical difficulty in completing the questionnaire.
Alternative methods of including the views of this group of users need to be considered. However, since the routine introduction of these questionnaires the response rates have risen and now range from $63 \%$ to $89 \%$ in the three centres.

Once patient satisfaction data are regularly generated, centres will be able to set local standards including minimum standards (below which patient satisfaction should not be allowed to fall) and target standards (the level of patient satisfaction which should be regularly achieved). Monitoring patient satisfaction consists of routinely comparing current results against these standards. The introduction of a measure of one aspect of the quality of care which is available on a regular basis is an important step forward.

The facility to make comparisons lies at the heart of any audit programme. The system described here allows such comparisons. Incorporation of PATSAT system into clinical audit (Committee on Clinical Audit Rehabilitation Medicine, disablement services annual report, April 1992) will provide information to help managers and health professionals to look for gaps in service provision and areas for improvements. Not only does it provide a framework for improving quality within the provider unit it is also a mechanism which purchasers may require, and pay for, to ensure the quality of services commissioned.

This system has now been integrated into the routine operation of 11 disablement services centres. Local and national user groups have been kept informed of the developments of PATSAT. The patient satisfaction sysem has a facility to explore topics in greater depth, and this has been undertaken for comfort of the limb. Future uses of these data include standard setting for each of the topics included in the questionnaire and monitoring the 
subsequent achievement of increased quality of service. The topics that are monitored may be increased or changed and, of course, as standards are achieved the target standard may be raised.

1 Larsen DE, Rootman I. Physicians' role performance and patient satisfaction. Soc Sci Med 1976;10:29-32.

2 Green J. On the receiving end. Health Services fournal 4 Aug 1988:880-1.
Smith C. Validatation of a patient satisfaction system in the United Kingdom. Qual Assur Health Care 1992;4:171-7.

Kerruish A, Wickings I, Tarrant P. Information from patients as a management tool: empowering managers to improve the quality of care. Hospital and Health Services Review April 1988.

5 Wickings I, Crown J, Harvey J, Kerruish A. Proof of th pudding. Health Services fournal, 31 Aug 1987: 1070-1.

6 Brent Health District. Effect of presenting management information to clinically accountable teams. Brent: Bren Health District, 1975. (Available from Health Information Unit and Management Research Team, Brent Health District.) 